\title{
A GeV to TeV view of shell-type SNRs
}

\section{Henrike Fleischhack* on behalf of the Fermi Large Area Telescope Collaboration and on behalf of the HAWC Collaboration \\ (a complete list of authors can be found at the end of the proceedings)}

Catholic University of America, Department of Physics

620 Michigan Ave. N.E., Washington, DC 20064, USA

NASA Goddard Space Flight Center,

8800 Greenbelt Rd, Greenbelt, MD 20771, USA

Center for Research and Exploration in Space Science and Technology,

8800 Greenbelt Rd, Greenbelt, MD 20771, USA

E-mail: fleischhack@cua.edu

Shock acceleration by the shells of supernova remnants (SNRs) has been hypothesized to be the mechanism that produces the bulk of Galactic Cosmic Rays, possibly up to PeV energies. Some SNRs have been shown to accelerate cosmic rays to TeV energies and above. But which SNRs are indeed efficient accelerators of protons and nuclei? And what is the maximum energy up to which they can efficiently accelerate particles? Measurements of non-thermal emission, especially in the gamma-ray regime, are essential to answer these questions.

The High-Altitude Water Cherenkov (HAWC) observatory, surveying the northern TeV gammaray sky, is currently the most sensitive wide field-of-view survey instrument in the VHE (veryhigh-energy, $>100 \mathrm{GeV}$ ) range and has recorded more than five years of data. The Large Area Telescope (LAT) onboard the Fermi satellite has been surveying the GeV gamma-ray sky for more than ten years. Combining measurements from both instruments allows the study of gamma-ray emission from SNRs over many orders of magnitude in energy. In this presentation, I will show measurements of VHE gamma-ray emission from Fermi-LAT-detected SNRs with the HAWC Observatory.

$37^{\text {th }}$ International Cosmic Ray Conference (ICRC 2021)

July 12 th - 23rd, 2021

Online - Berlin, Germany

\footnotetext{
${ }^{*}$ Presenter
} 


\section{Introduction}

Shell-type supernova remnants (SNRs) have been considered as potential sources of Galactic cosmic rays due to two main arguments (see e.g. [1] and references therein). First, diffusive shock acceleration provides a mechanism for the expanding SNR shell to accelerate charged particles to relativistic speeds. Second, given the estimated rate of supernova explosions in the galaxy, the average energy released, and the predicted acceleration efficiencies of SNRs, these objects are expected to have sufficient power to sustain the measured intensity of Galactic cosmic rays.

In particular, young SNRs (hundreds of years old) are thought to be efficient accelerators of cosmic rays. Middle-aged SNRs (up to tens of thousands of years) can still be surrounded by a population of previously accelerated cosmic rays. However, it is unclear if SNRs do indeed have the ability to accelerate particles up to the so-called "knee", a break in the cosmic-ray energy spectrum around a few $\mathrm{PeV}$, which is thought to indicate the maximum energy in the Galactic component of cosmic rays.

Any SNR that accelerates cosmic-ray protons to relativistic energies should also produce nonthermal gamma-ray emission due to interactions of these protons with the interstellar medium in/near the remnant. These interactions produce (among other things) neutral pions, which in turn decay into gamma rays. This emission mechanism is referred to as hadronic emission. The gammaray energy spectrum is predicted to follow the underlying cosmic-ray energy spectrum. Any feature in the cosmic ray spectrum such as a break or cutoff should also be seen in gamma rays, at slightly lower energies. Thus, we can use the gamma-ray energy spectrum from $\mathrm{GeV}$ s to hundreds of $\mathrm{TeV}$ to investigate cosmic-ray acceleration up to PeV energies in SNRs.

SNRs accelerating electrons to relativistic energies are also expected to produce non-thermal gamma-ray emission due to inverse Compton scattering of the CMB (or in some cases, ambient infrared or optical photon fields) and/or Bremsstrahlung processes in the presence of matter. These electrons are also expected to emit Synchrotron radiation at lower wavelengths (radio to X-ray, depending on the ambient magnetic fields). These emission mechanisms are referred to as leptonic emission. In fact, most known SNR shells have been detected in radio surveys.

HAWC is a large-field-of-view gamma-ray observatory sensitive in the energy range from hundreds of $\mathrm{GeV}$ to hundreds of $\mathrm{TeV}$. HAWC has been surveying the northern $\mathrm{TeV}$ gamma-ray sky since 2015 and in its latest catalog reports 65 gamma-ray sources [2].

The Large Area Telescope (LAT) aboard the Fermi satellite has been surveying the gamma-ray sky in the energy range from tens of $\mathrm{MeV}$ to $2 \mathrm{TeV}$ for more than ten years now. The 10-year Fermi-LAT data release (4FGL-DR2, [3]) contains 24 firmly identified shell-type SNRs and an additional 19 sources that are less firmly associated with SNRs, as well as 96 sources classified as "spp" type, indicating potential association with a PWN and/or SNR. At least three SNRs (IC 443, W44, and W51C) show a significant spectral feature, the "pion bump" at around $100 \mathrm{MeV}$, indicating that the gamma-ray emission is dominated by hadronic processes [4, 5]. However, for many other SNRs, it is currently not clear if the gamma-ray emission is dominated by hadronic or leptonic processes.

Several SNRs have also been detected at TeV energies. As of 2021/02/16, TeVCat ${ }^{1}$, a listing of $\mathrm{TeV}$ gamma-ray sources seen by HAWC and other ground-based gamma-ray observatories such as

\footnotetext{
${ }^{1}$ http://tevcat. uchicago.edu/
} 


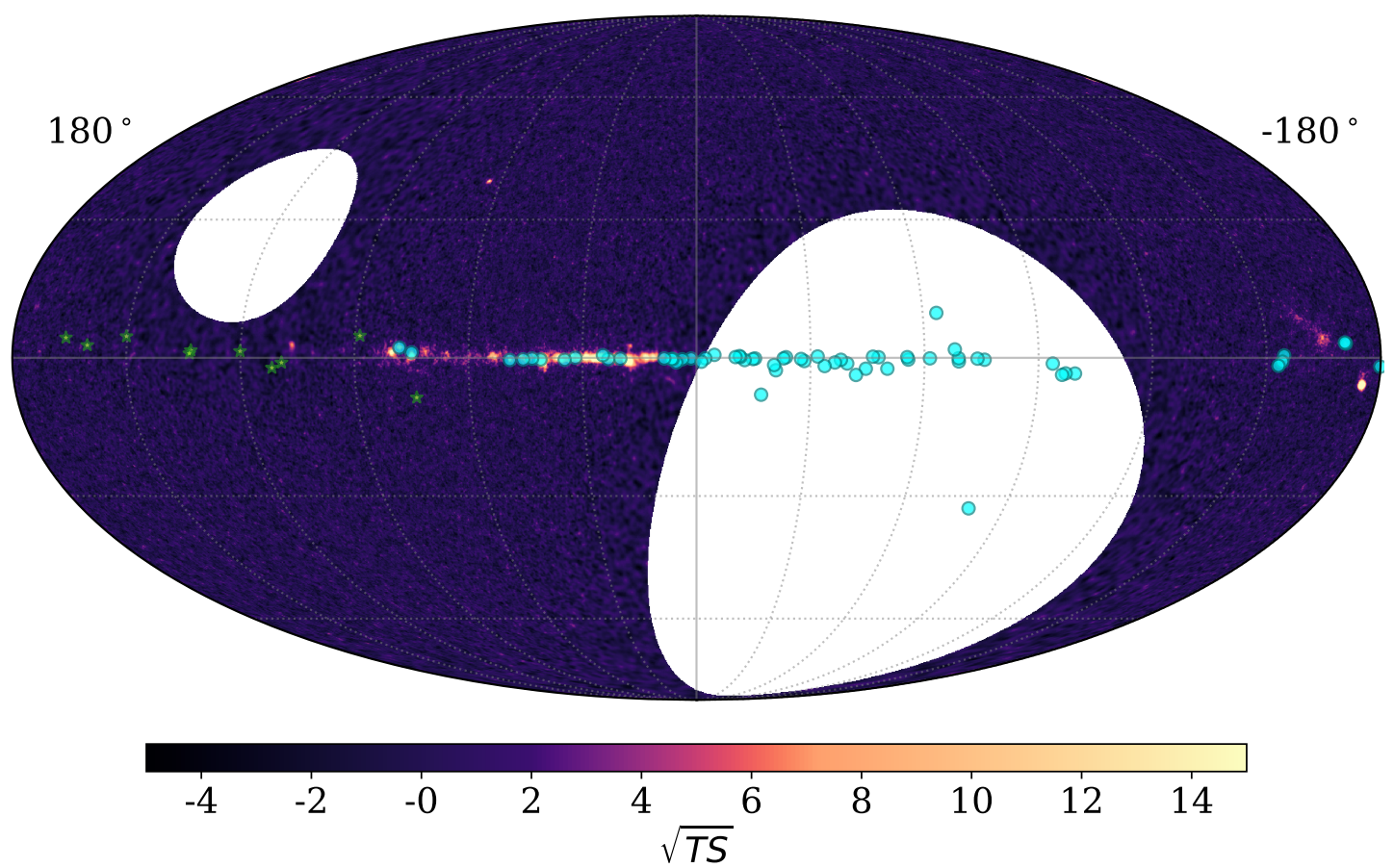

Figure 1: HAWC's all-sky significance map for the point source search, based on 1523 days of observation, in Galactic coordinates [2]. White areas of the sky are not observed by HAWC. Green stars mark the position of shell-type SNRs and SNR candidates from the LAT catalogs as described in Section 2.1. Aqua circles mark SNRs and SNR candidates that do not pass the selection criteria (outside of HAWC's field of view or overlapping with significant gamma-ray emission seen by HAWC).

the imaging air-Cherenkov telescopes H.E.S.S., MAGIC, and VERITAS, lists 16 VHE gamma-ray sources associated with shell-type SNRs and 11 sources ascribed to the interaction of SNRs with nearby molecular clouds. However, many of these sources show spectral softening or a cutoff between $\mathrm{GeV}$ and $\mathrm{TeV}$ energies, indicating that those SNRs may not be able to accelerate cosmic rays up to $\mathrm{PeV}$ energies.

Here, we perform a search for TeV gamma-ray emission from $\mathrm{GeV}$-emitting SNRs, focussing on SNRs that are not significantly detected by HAWC. Upper limits on the TeV gamma-ray flux will be determined. For SNRs where the upper limits are below the extrapolation from the $\mathrm{GeV}$ gamma-ray spectrum, we will have shown the presence of a break or cutoff in the spectrum, and can relate upper limits on the cutoff energy in the gamma-ray spectrum to the cutoff energy in the underlying proton or electron spectrum.

This contribution describes the source selection and analysis method; full results will be provided in a separate, peer-reviewed paper (currently under preparation). 


\section{Data and Analysis Methods}

\subsection{Source Selection}

GeV-detected shell-type supernova remnants (SNRs) and SNR candidates were selected from the following four catalogs released by the Fermi-LAT collaboration:

- The LAT 10-year Source Catalog (4FGL-DR2, [3, 6]),

- The Third Fermi-LAT Catalog of High-Energy Sources (3FHL, [7]),

- Extended Sources in the Galactic Plane (FGES, [8]), and

- The First LAT Supernova Remnant Catalog (1SC, [9]).

Sources were selected from these catalogs as follows:

- 1SC sources with the "classification" property given as "classified" (meaning, classified as SNRs).

- 4FGL-DR2 and 3FHL sources with source type "SNR" (firmly identified as a shell-type SNR) or "snr" (less firmly associated with a shell-type SNR).

- 3FHL sources associated with a 4FGL-DR2 SNR/snr source (via the "ASSOC_FHL" property)

- FGES sources not associated with a known PWN, given the associations listed in the FGES paper and the source types in the 3FHL, 4FGL-DR2, and TeVCat ${ }^{2}$.

- 3FHL and 4FGL-DR2 sources of type "spp" 3 within $0.3^{\circ}$ of a previously selected FGES or $1 \mathrm{SC}$ source.

Sources were required to have declinations (J2000) between $-25^{\circ}$ and $65^{\circ}$ to lie within HAWC's field of view.

Using the HAWC significance maps upon which the 3HWC catalog [2] was based, SNRs were selected that did not overlap with regions where HAWC detects significant gamma-ray emission.

As all four LAT catalogs used different datasets and different energy ranges, a single SNR may be detected in only a subset of the catalogs. Additionally, SNRs detected in multiple catalogs may be found at slightly different locations or with different morphologies. Sources within $0.3^{\circ}$ of each other were considered to be in the same "region" if their extensions were also similar, which each region likely corresponding to the same physical source.

Table 1 shows the ten selected SNRs and SNR candidates. Several are found in more than one catalog, leading to 21 sources to be studied.

\subsection{HAWC Data analysis}

\subsubsection{Dataset and Analysis Software}

The analysis presented here is based on 1523 days of data recorded by HAWC. The same dataset was used for 3HWC, the third HAWC catalog. More details about the dataset can be found in [2].

HAWC-internal software was used for data reduction (calibration, event reconstruction, background rejection, and binning). The multi-mission maximum likelihood (3ML) framework [10] with the HAWC accelerated likelihood plugin (HAL) [11] was used to analyze the binned data.

\footnotetext{
${ }^{2} \mathrm{http}: / /$ tevcat.uchicago.edu

3"Sources of unknown nature but overlapping with known SNRs or PWNe and thus candidates to these classes."
} 
Table 1: SNRs and SNR candidates selected as described in Section 2.1. RA and Dec are the Right Ascension and Declination of the source center (J2000 epoch). Ext is the exension (radius for sources modeled with a disk morphology, approximate radius or semi-major axis of a circle or ellipse covering the emission region for sources with irregular morphologies). Thin lines delineated between "regions" (e.g., the first three sources are all presumed to be associated with the same physical object).

\begin{tabular}{llrrrlr}
\hline Name & Association & RA $\left[^{\circ}\right]$ & Dec $\left[^{\circ}\right]$ & Ext. $\left[^{\circ}\right]$ & Shape & $\pm \sqrt{T S}$ \\
\hline 3FHL J2051.0+3040e & Cygnus Loop & 312.75 & 30.67 & 1.65 & CygnusLoop.fits & 0.60 \\
4FGL J2051.0+3049e & Cygnus Loop & 312.75 & 30.83 & 1.65 & CygnusLoop.fits & 0.44 \\
SNR074.0-08.5 & & 312.77 & 30.90 & 1.74 & Disk & 0.66 \\
\hline SNR089.0+04.7 & & 311.15 & 50.42 & 0.97 & Disk & -0.07 \\
4FGL J2045.2+5026e & HB 21 & 311.32 & 50.44 & 1.19 & Disk & -1.34 \\
\hline SNR109.1-01.0 & & 345.41 & 58.83 & 0.00 & & -0.41 \\
4FGL J2301.9+5855e & CTB 109 & 345.49 & 58.92 & 0.25 & Disk & -0.50 \\
3FHL J2301.9+5855e & CTB 109 & 345.49 & 58.92 & 0.25 & Disk & -0.50 \\
FGES J2302.0+5855 & & 345.49 & 58.92 & 0.25 & Disk & -0.50 \\
\hline SNR111.7-02.1 & & 350.85 & 58.83 & 0.00 & & 0.46 \\
4FGL J2323.4+5849 & Cas A & 350.86 & 58.82 & 0.00 & & -0.45 \\
3FHL J2323.4+5848 & Cassiopeia A & 350.87 & 58.82 & 0.00 & & 0.44 \\
\hline 4FGL J0025.3+6408 & Tycho & 6.34 & 64.15 & 0.00 & & -1.21 \\
3FHL J0025.5+6407 & Tycho & 6.38 & 64.13 & 0.00 & & -1.20 \\
\hline 4FGL J0221.4+6241e & HB 3 & 35.36 & 62.69 & 0.80 & Disk & -0.01 \\
\hline 4FGL J0222.4+6156e & W 3 & 35.62 & 61.94 & 0.60 & W3.fits & -0.52 \\
\hline 4FGL J0427.2+5533e & SNR G150.3+04.5 & 66.82 & 55.55 & 1.51 & Disk & -0.22 \\
3FHL J0427.2+5533e & SNR G150.3+4.5 & 66.82 & 55.55 & 1.51 & Disk & 1.10 \\
FGES J0427.2+5533 & & 66.82 & 55.55 & 1.52 & Disk & 1.11 \\
\hline 4FGL J0500.3+4639e & HB 9 & 75.08 & 46.66 & 1.00 & HB9.fits & -1.28 \\
\hline 4FGL J0526.7+4254 & SNR G166.0+04.3 & 81.69 & 42.92 & 0.00 & & 0.00 \\
\hline
\end{tabular}

Each source was fit separately, with a region of interest (ROI) centered on the source and with a radius of $5^{\circ}$ for point sources, $5^{\circ}+$ source extension (see Table 1 ) for extended sources.

\subsubsection{Source Modeling}

Each source was modeled according to the position and morphology (disk or external template) given in the relevant LAT catalog. All parameters related to the morphology were kept fixed in the analysis.

For the spectrum, two separate analyses were carried out (both using the spatial model as described above):

1. The spectral shape was modeled according to the relevant LAT catalog, with free normalization. A normalization parameter $s$ was introduced which scales the flux normalization relative to the extrapolation from the LAT energies.

2. The spectral shape was modeled according to the relevant LAT catalog, multiplied by an 


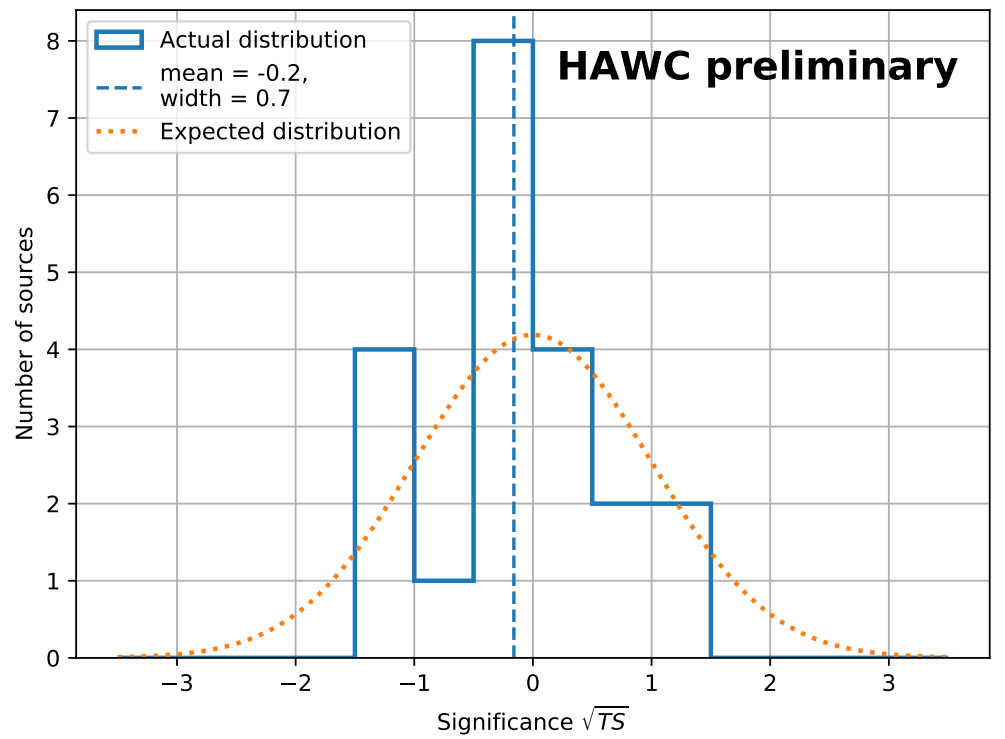

Figure 2: Distribution of the detection significances from Table 1. The expected distribution is a Gaussian distribution with mean 0 and width 1 .

exponential cutoff $\exp \left(-\frac{E}{E_{c}}\right)$ with free cutoff energy $E_{c}$. In this case, all other parameters including the normalization were kept fixed.

A forward-fold likelihood fit (see [10]) was performed to find the best-fit values of the normalization constant $s$ or the cutoff energy $E_{c}$. For the first case, we also determined the test statistic $T S=-2 \ln \left(\frac{L_{0}}{L_{S}}\right)$, where $L_{S}$ is the value of the likelihood at the best-fit value of $s$ and $L_{0}$ is the value of the likelihood without the source in question (i.e., with $s=0$ ). $\pm \sqrt{T S}$ then corresponds to the detection significance of a given source, where we chose the negative sign if and only if $s<0$.

\subsubsection{Upper Limit Determination}

As none of these sources were detected by HAWC, the most we can do is to set upper limits on the flux normalization. If the upper limit on $s$ is above 1, that would mean that the non-detection of the given source is compatible with the extrapolation of the $\mathrm{GeV}$ spectrum to $\mathrm{TeV}$ energies (meaning, the source could be just too weak for HAWC to be able to see it) and we are not able to constrain its spectrum further. However, for sources with an upper limit on $s$ below one, we can conclude that the HAWC non-detection is inconsistent with the extrapolation of the $\mathrm{GeV}$ spectrum to $\mathrm{TeV}$ energies - meaning that the spectrum must have a break or cutoff at $\mathrm{TeV}$ energies or above. We can try to set an upper limit on such a cutoff.

In both cases (upper limit on the normalization scale factor $s$ and upper limit on the cutoff energy $E_{c}$ ), the limits were obtained from the likelihood profile. $90 \%$ confidence level upper limits correspond to a 3.28 increase in the log-likelihood. 


\subsubsection{Systematic Uncertainties}

The systematic uncertainties due to the modeling of the HAWC detector were taken into account as described in [2], with the addition of the systematic uncertainty due to the source spectrum assumed in the preparation of the detector response file. For point sources, we also accounted for HAWC's systematic pointing uncertainty by letting the source positions float within the uncertainty and profiling over the source position when calculating the upper limit.

\section{Results}

Figure 2 shows the significance distribution of 2 . The distribution is compatible with a Gaussian distribution with mean 0 and width 1 as expected from the "background-only" case, indicating that there is no evidence for sub-threshold $\mathrm{TeV}$ emission in the selected sample of GeV-detected SNRs and SNR candidates.

\section{Outlook}

Full results including upper limits on the $\mathrm{TeV}$ emission from $\mathrm{GeV}$ detected SNRs will be presented in a dedicated publication (under preparation).

\section{References}

[1] J.W. Hewitt and M. Lemoine-Goumard, Observations of supernova remnants and pulsar wind nebulae at gamma-ray energies, C R Phys 16 (2015) 674.

[2] A. Albert et al., 3HWC: The Third HAWC Catalog of Very-high-energy Gamma-Ray Sources, ApJ 905 (2020) 76.

[3] S. Abdollahi et al., Fermi Large Area Telescope Fourth Source Catalog, ApJS 247 (2020) 33.

[4] M. Ackermann et al., Detection of the characteristic pion-decay signature in supernova remnants, Science 339 (2013) 807-811.

[5] T. Jogler and S. Funk, REVEALING W5IC AS A COSMIC RAY SOURCE USING FERMI-LAT DATA, The Astrophysical Journal 816 (2016) 100.

[6] J. Ballet, T.H. Burnett, S.W. Digel and B. Lott, "Fermi Large Area Telescope Fourth Source Catalog Data Release 2." fermi.gsfc.nasa.gov/ssc/data/access/lat/10yr_catalog, 2020.

[7] M. Ajello et al., 3FHL: The Third Catalog of Hard Fermi-LAT Sources, ApJS 232 (2017) 18.

[8] M. Ackermann et al., Search for Extended Sources in the Galactic Plane Using Six Years of Fermi-Large Area Telescope Pass 8 Data above 10 GeV, ApJ 843 (2017) 139.

[9] F. Acero et al., THE FIRST FERMI LAT SUPERNOVA REMNANT CATALOG, ApJS 224 (2016) 8. 
[10] G. Vianello et al., The Multi-Mission Maximum Likelihood framework (3ML), in Proc. 34th ICRC, PoS, 2015, https://inspirehep.net/record/1385718/files/arXiv:1507.08343.pdf [1507.08343].

[11] C. Brisbois et al., Characterizing gamma-ray sources with HAL (HAWC Accelerated likelihood) and 3ML, in these proceedings, 2021.

\section{Acknowledgments}

We acknowledge the support from: the US National Science Foundation (NSF); the US Department of Energy Office of High-Energy Physics; the Laboratory Directed Research and Development (LDRD) program of Los Alamos National Laboratory; Consejo Nacional de Ciencia y Tecnología (CONACyT), México, grants 271051, 232656, 260378, 179588, 254964, 258865, 243290, 132197 , A1-S-46288, A1-S-22784, cátedras 873, 1563, 341, 323, Red HAWC, México; DGAPA-UNAM grants IG101320, IN111716-3, IN111419, IA102019, IN110621, IN110521; VIEP-BUAP; PIFI 2012, 2013, PROFOCIE 2014, 2015; the University of Wisconsin Alumni Research Foundation; the Institute of Geophysics, Planetary Physics, and Signatures at Los Alamos National Laboratory; Polish Science Centre grant, DEC-2017/27/B/ST9/02272; Coordinación de la Investigación Científica de la Universidad Michoacana; Royal Society - Newton Advanced Fellowship 180385; Generalitat Valenciana, grant CIDEGENT/2018/034; Chulalongkorn University's CUniverse (CUAASC) grant; Coordinación General Académica e Innovación (CGAI-UdeG), PRODEP-SEP UDG-CA499; Institute of Cosmic Ray Research (ICRR), University of Tokyo, H.F. acknowledges support by NASA under award number 80GSFC21M0002. We also acknowledge the significant contributions over many years of Stefan Westerhoff, Gaurang Yodh and Arnulfo Zepeda Dominguez, all deceased members of the HAWC collaboration. Thanks to Scott Delay, Luciano Díaz and Eduardo Murrieta for technical support. 


\section{Full Authors List: HAWC Collaboration}

A.U. Abeysekara ${ }^{48}$, A. Albert ${ }^{21}$, R. Alfaro ${ }^{14}$, C. Alvarez ${ }^{41}$, J.D. Álvarez ${ }^{40}$, J.R. Angeles Camacho ${ }^{14}$, J.C. Arteaga-Velázquez ${ }^{40}$, K. P. Arunbabu $^{17}$, D. Avila Rojas ${ }^{14}$, H.A. Ayala Solares ${ }^{28}$, R. Babu ${ }^{25}$, V. Baghmanyan ${ }^{15}$, A.S. Barber ${ }^{48}$, J. Becerra Gonzalez ${ }^{11}$, E. BelmontMoreno $^{14}$, S.Y. BenZvi ${ }^{29}$, D. Berley ${ }^{39}$, C. Brisbois ${ }^{39}$, K.S. Caballero-Mora ${ }^{41}$, T. Capistrán ${ }^{12}$, A. Carramiñana ${ }^{18}$, S. Casanova ${ }^{15}$, O. Chaparro-Amaro $^{3}$, U. Cotti ${ }^{40}$, J. Cotzomi ${ }^{8}$, S. Coutiño de León ${ }^{18}$, E. De la Fuente ${ }^{46}$, C. de León ${ }^{40}$, L. Diaz-Cruz ${ }^{8}$, R. Diaz Hernandez ${ }^{18}$, J.C. Díaz-Vélez ${ }^{46}$, B.L. Dingus ${ }^{21}$, M. Durocher ${ }^{21}$, M.A. DuVernois ${ }^{45}$, R.W. Ellsworth ${ }^{39}$, K. Engel ${ }^{39}$, C. Espinoza ${ }^{14}$, K.L. Fan ${ }^{39}$, K. Fang $^{45}$, M. Fernández Alonso ${ }^{28}$, B. Fick ${ }^{25}$, H. Fleischhack ${ }^{51,11,52}$, J.L. Flores ${ }^{46}$, N.I. Fraija ${ }^{12}$, D. Garcia ${ }^{4}$, J.A. García-González ${ }^{20}$, J. L. García-Luna ${ }^{46}$, G. García-Torales ${ }^{46}$, F. Garfias ${ }^{12}$, G. Giacinti ${ }^{22}$, H. Goksu ${ }^{22}$, M.M. González ${ }^{12}$, J.A. Goodman ${ }^{39}$, J.P. Harding ${ }^{21}$, S. Hernandez ${ }^{14}$, I. Herzog ${ }^{25}$, J. Hinton ${ }^{22}$, B. Hona ${ }^{48}$, D. Huang ${ }^{25}$, F. Hueyotl-Zahuantitla ${ }^{41}$, C.M. Hui ${ }^{23}$, B. Humensky ${ }^{39}$, P. Hüntemeyer ${ }^{25}$, A. Iriarte ${ }^{12}$, A. Jardin-Blicq ${ }^{22,49,50}$, H. Jhee ${ }^{43}$, V. Joshi ${ }^{7}$, D. Kieda ${ }^{48}$, G J. Kunde ${ }^{21}$, S. Kunwar ${ }^{22}$, A. Lara ${ }^{17}$, J. Lee ${ }^{43}$, W.H. Lee ${ }^{12}$, D. Lennarz ${ }^{9}$, H. León Vargas ${ }^{14}$, J. Linnemann ${ }^{24}$, A.L. Longinotti ${ }^{12}$, R. López-Coto ${ }^{19}$, G. Luis-Raya ${ }^{44}$, J. Lundeen ${ }^{24}$, K. Malone ${ }^{21}$, V. Marandon $^{22}$, O. Martinez ${ }^{8}$, I. Martinez-Castellanos ${ }^{39}$, H. Martínez-Huerta ${ }^{38}$, J. Martínez-Castro ${ }^{3}$, J.A.J. Matthews ${ }^{42}$, J. McEnery ${ }^{11}$, P. Miranda-Romagnoli $^{34}$, J.A. Morales-Soto ${ }^{40}$, E. Moreno ${ }^{8}$, M. Mostafá ${ }^{28}$, A. Nayerhoda ${ }^{15}$, L. Nellen ${ }^{13}$, M. Newbold ${ }^{48}$, M.U. Nisa ${ }^{24}$, R. Noriega-Papaqui ${ }^{34}$, L. Olivera-Nieto ${ }^{22}$, N. Omodei ${ }^{32}$, A. Peisker ${ }^{24}$, Y. Pérez Araujo ${ }^{12}$, E.G. Pérez-Pérez ${ }^{44}$, C.D. Rho ${ }^{43}$, C. Rivière ${ }^{39}$, D. Rosa-Gonzalez ${ }^{18}$, E. Ruiz-Velasco ${ }^{22}$, J. Ryan ${ }^{26}$, H. Salazar ${ }^{8}$, F. Salesa Greus ${ }^{15,53}$, A. Sandoval ${ }^{14}$, M. Schneider ${ }^{39}$, H. Schoorlemmer ${ }^{22}$, J. Serna-Franco ${ }^{14}$, G. Sinnis ${ }^{21}$, A.J. Smith ${ }^{39}$, R.W. Springer ${ }^{48}$, P. Surajbali ${ }^{22}$, I. Taboada ${ }^{9}$, M. Tanner ${ }^{28}$, K. Tollefson ${ }^{24}$, I. Torres ${ }^{18}$, R. Torres-Escobedo $^{30}$, R. Turner ${ }^{25}$, F. Ureña-Mena ${ }^{18}$, L. Villaseñor ${ }^{8}$, X. Wang ${ }^{25}$, I.J. Watson ${ }^{43}$, T. Weisgarber ${ }^{45}$, F. Werner ${ }^{22}$, E. Willox ${ }^{39}$, J. Wood ${ }^{23}$, G.B. Yodh ${ }^{35}$, A. Zepeda ${ }^{4}$, H. Zhou ${ }^{30}$

${ }^{1}$ Barnard College, New York, NY, USA, ${ }^{2}$ Department of Chemistry and Physics, California University of Pennsylvania, California, PA, USA, ${ }^{3}$ Centro de Investigación en Computación, Instituto Politécnico Nacional, Ciudad de México, México, ${ }^{4}$ Physics Department, Centro de Investigación y de Estudios Avanzados del IPN, Ciudad de México, México, ${ }^{5}$ Colorado State University, Physics Dept., Fort Collins, CO, USA, ${ }^{6}$ DCI-UDG, Leon, Gto, México, ${ }^{7}$ Erlangen Centre for Astroparticle Physics, Friedrich Alexander Universität, Erlangen, BY, Germany, ${ }^{8}$ Facultad de Ciencias Físico Matemáticas, Benemérita Universidad Autónoma de Puebla, Puebla, México, ${ }^{9}$ School of Physics and Center for Relativistic Astrophysics, Georgia Institute of Technology, Atlanta, GA, USA, ${ }^{10}$ School of Physics Astronomy and Computational Sciences, George Mason University, Fairfax, VA, USA, ${ }^{11}$ NASA Goddard Space Flight Center, Greenbelt, MD, USA, ${ }^{12}$ Instituto de Astronomía, Universidad Nacional Autónoma de México, Ciudad de México, México, ${ }^{13}$ Instituto de Ciencias Nucleares, Universidad Nacional Autónoma de México, Ciudad de México, México, ${ }^{14}$ Instituto de Física, Universidad Nacional Autónoma de México, Ciudad de México, México, ${ }^{15}$ Institute of Nuclear Physics, Polish Academy of Sciences, Krakow, Poland, ${ }^{16}$ Instituto de Física de São Carlos, Universidade de São Paulo, São Carlos, SP, Brasil, ${ }^{17}$ Instituto de Geofísica, Universidad Nacional Autónoma de México, Ciudad de México, México, ${ }^{18}$ Instituto Nacional de Astrofísica, Óptica y Electrónica, Tonantzintla, Puebla, México, ${ }^{19}$ INFN Padova, Padova, Italy, ${ }^{20}$ Tecnologico de Monterrey, Escuela de Ingeniería y Ciencias, Ave. Eugenio Garza Sada 2501, Monterrey, N.L., 64849, México, ${ }^{21}$ Physics Division, Los Alamos National Laboratory, Los Alamos, NM, USA, ${ }^{22}$ Max-Planck Institute for Nuclear Physics, Heidelberg, Germany, ${ }^{23}$ NASA Marshall Space Flight Center, Astrophysics Office, Huntsville, AL, USA, ${ }^{24}$ Department of Physics and Astronomy, Michigan State University, East Lansing, MI, USA, ${ }^{25}$ Department of Physics, Michigan Technological University, Houghton, MI, USA, ${ }^{26}$ Space Science Center, University of New Hampshire, Durham, NH, USA, ${ }^{27}$ The Ohio State University at Lima, Lima, OH, USA, ${ }^{28}$ Department of Physics, Pennsylvania State University, University Park, PA, USA, ${ }^{29}$ Department of Physics and Astronomy, University of Rochester, Rochester, NY, USA, ${ }^{30}$ Tsung-Dao Lee Institute and School of Physics and Astronomy, Shanghai Jiao Tong University, Shanghai, China, ${ }^{31}$ Sungkyunkwan University, Gyeonggi, Rep. of Korea, ${ }^{32}$ Stanford University, Stanford, CA, USA, ${ }^{33}$ Department of Physics and Astronomy, University of Alabama, Tuscaloosa, AL, USA, ${ }^{34}$ Universidad Autónoma del Estado de Hidalgo, Pachuca, Hgo., México, ${ }^{35}$ Department of Physics and Astronomy, University of California, Irvine, Irvine, CA, USA, ${ }^{36}$ Santa Cruz Institute for Particle Physics, University of California, Santa Cruz, Santa Cruz, CA, USA, ${ }^{37}$ Universidad de Costa Rica, San José, Costa Rica, ${ }^{38}$ Department of Physics and Mathematics, Universidad de Monterrey, San Pedro Garza García, N.L., México, ${ }^{39}$ Department of Physics, University of Maryland, College Park, MD, USA, ${ }^{40}$ Instituto de Física y Matemáticas, Universidad Michoacana de San Nicolás de Hidalgo, Morelia, Michoacán, México, ${ }^{41}$ FCFM-MCTP, Universidad Autónoma de Chiapas, Tuxtla Gutiérrez, Chiapas, México, ${ }^{42}$ Department of Physics and Astronomy, University of New Mexico, Albuquerque, NM, USA, ${ }^{43}$ University of Seoul, Seoul, Rep. of Korea, ${ }^{44}$ Universidad Politécnica de Pachuca, Pachuca, Hgo, México, ${ }^{45}$ Department of Physics, University of Wisconsin-Madison, Madison, WI, USA, ${ }^{46}$ CUCEI, CUCEA, Universidad de Guadalajara, Guadalajara, Jalisco, México, ${ }^{47}$ Universität Würzburg, Institute for Theoretical Physics and Astrophysics, Würzburg, Germany, ${ }^{48}$ Department of Physics and Astronomy, University of Utah, Salt Lake City, UT, USA, ${ }^{49}$ Department of Physics, Faculty of Science, Chulalongkorn University, Pathumwan, Bangkok 10330, Thailand, ${ }^{50}$ National Astronomical Research Institute of Thailand (Public Organization), Don Kaeo, MaeRim, Chiang Mai 50180, Thailand, ${ }^{51}$ Department of Physics, Catholic University of America, Washington, DC, USA, ${ }^{52}$ Center for Research and Exploration in Space Science and Technology, NASA/GSFC, Greenbelt, MD, USA, ${ }^{53}$ Instituto de Física Corpuscular, CSIC, Universitat de València, Paterna, Valencia, Spain

\section{No full author list for the Fermi-LAT collaboration is provided here in accordance with LAT collaboration policy for conference proceedings.}

